\title{
The Rationale, Feasibility, and Optimal Training of the Non-Physician Medical Nutrition Scientist
}

\author{
Susan E. Ettinger, ${ }^{1}$ Jennifer A. Nasser, ${ }^{1,2}$ Ellen S. Engelson, ${ }^{1}$ Jeanine B. Albu, ${ }^{1}$ \\ Sami Hashim, ${ }^{1}$ and F. Xavier Pi-Sunyer ${ }^{1}$ \\ ${ }^{1}$ New York Obesity Nutrition Research Center, Columbia University, New York, NY 10032, USA \\ ${ }^{2}$ Nutrition Sciences, Drexel University, Philadelphia, PA 19104, USA \\ Correspondence should be addressed to F. Xavier Pi-Sunyer; fxp1@columbia.edu
}

Received 4 December 2014; Accepted 13 January 2015

Academic Editor: Caryl Nowson

Copyright (C) 2015 Susan E. Ettinger et al. This is an open access article distributed under the Creative Commons Attribution License, which permits unrestricted use, distribution, and reproduction in any medium, provided the original work is properly cited.

\begin{abstract}
Dietary components have potential to arrest or modify chronic disease processes including obesity, cancer, and comorbidities. However, clinical research to translate mechanistic nutrition data into clinical interventions is needed. We have developed a oneyear transitional postdoctoral curriculum to prepare nutrition scientists in the language and practice of medicine and in clinical research methodology before undertaking independent research. Candidates with an earned doctorate in nutrition science receive intensive, didactic training at the interface of nutrition and medicine, participate in supervised medical observerships, and join ongoing clinical research. To date, we have trained four postdoctoral fellows. Formative evaluation revealed several learning barriers to this training, including deficits in prior medical science knowledge and diverse perceptions of the role of the translational nutrition scientist. Several innovative techniques to address these barriers are discussed. We propose the fact that this "train the trainer" approach has potential to create a new translational nutrition researcher competent to identify clinical problems, collaborate with clinicians and researchers, and incorporate nutrition science across disciplines from "bench to bedside." We also expect the translational nutrition scientist to serve as an expert resource to the medical team in use of nutrition as adjuvant therapy for the prevention and management of chronic disease.
\end{abstract}

\section{Introduction}

The ideal training and scope of practice of nutrition professionals has been a bone of contention for as long as medicine has existed. No less a physician than Hippocrates [1] wrote over 2400 years ago, "Let food be thy medicine and medicine be thy food." This recommendation has been supported by abundant evidence suggesting that specific components in food can ameliorate deleterious effects of metabolic derangements [2], inflammation [3], and organ dysfunction in humans and animals $[4,5]$. Despite this suggestive evidence, and even in the face of widespread chronic disease and soaring medical costs, translation of these experimental findings into clinical trials to guide patient care has been limited [6].

Several questions must be addressed in clinical trials before targeted diet guidelines for humans can be established [7-9]. Areas in need of clarification include alteration in food composition due to production and processing methods, optimal nutrient dosage, bioavailability, interactions with other dietary components, and possible toxicity. Translation to actual patient care is further complicated by individual genetic profiles, differences in normal and transformed cell and tissue response to food components as a function of tissue specific receptors, and pathophysiologic changes due to concomitant diseases and chronological age. An additional layer of complexity results from the impact of food components on the vast microbiota in the gut, with multiple potential impacts on risk for cancer and chronic disease [10]. These concerns underscore the need to train a new nutrition professional with an understanding of the language and practice of medicine, competent to develop a nutrition-focused biological plausibility [11, 12], to solve clinical questions and to translate data into targeted nutrition guidelines for clinical care. 
Theoretically, translational clinical research in medical nutrition could be accomplished by the physician with specialized postgraduate training. In 1995, the Committee on Clinical Practice Issues in Health and Disease of the American Society for Clinical Nutrition proposed that the American Board of Nutrition certify the physician nutrition specialist (PNS) [13]. The PNS was envisioned as functioning in all facets of nutrition services, from complex clinical nutrition support issues to community health promotion programs, and was expected to be especially valuable in the academic setting as a role model and educator. An Intersociety Professional Nutrition Education Consortium (IPNEC) was founded to establish educational standards and a unified mechanism for PNS certification. Actual nutrition training required for the PNS is largely clinical. Candidates complete as little as 6 months of mentored clinical nutrition experience and formal instruction, either as a block or as an equivalent amount of time ( 1000 hours) integrated among other clinical duties over a longer time period. Approximately one-half of applicants indicated that they had completed some fellowship training in nutrition, either as stand-alone nutrition fellowships or combined with other subspecialties. As a result, in 2009, most of the 368 PNS diplomats surveyed indicated a career focus in clinical medicine, with few reporting a focus in clinical research [14]. The dearth of physicians who function as "pure" clinical nutritionists was described over 40 years ago by van Itallie [15] and continues to be of concern [16]. While PNS training creates physicians competent to provide medical nutrition care, it does not effectively create an academic professional whose primary focus is to identify a clinical problem, devise a mechanistic hypothesis to test the efficacy of targeted diet modification as adjuvant medical therapy, and conduct substantive translational clinical research.

McLaren [17] proposed that leadership in nutrition education and clinical research would be more effectively accomplished through collaboration between the PNS and a nutrition scientist who understands the language and practice of clinical medicine. Although the registered dietitian has been proposed as the ideal collaborator with the PNS [18], McLaren identified a clear distinction between nutrition and dietetics. He proposed that nutrition is an integral part of biology that deals with the physiological roles of food components, while the goal of dietetics is social: "to maintain or improve health through optimal feeding practices" [19]. We propose that the ideal collaborator must be a doctoral level nutrition scientist, cross-trained in the language and practice of medicine and competent to conduct clinical research. The Medical Nutrition Scientist (MNS) would also function as an educator and researcher and be in an ideal position to liaise with the physician and basic scientist to design targeted clinical studies to improve patient care.

\section{Methods}

Theoretically, the candidate for MNS training must have doctoral level expertise in food and nutrition science as set forth by the American Society for Nutrition [20]. This knowledge base encompasses clear understanding of food as a source of nutrients, bioavailability of nutrients and nonnutrient food components, risk from toxins and carcinogens in the diet, and the impact of food processing methods that modify diet components. The MNS must have a working knowledge of dietary factors that regulate whole body nutrient homeostasis and the composition and function of the gut microbiota. In addition, the MNS must have expert knowledge of the actions of the nutrients at the cellular and molecular levels.

Doctoral training in nutrition science is based on undergraduate prerequisites similar to those required for medical training: one year of physics, one year of biology, one year of English, and two years of chemistry through organic chemistry (prerequisites as specified by the Association of American Medical Colleges https://www.aamc.org/ students/applying/requirements/). Expertise in nutrition science is achieved through graduate courses that focus on the molecular and cellular biochemistry of foods and nutrients, their relationship to human physiology, and mechanisms of homeostatic regulation. Building on expertise developed in doctoral training, MNS training must integrate nutrition and medicine to develop understanding of (1) mechanisms of disease etiology, pathology, and morphologic signs and symptoms together with nutrient actions that target these processes, (2) current medical techniques for diagnostic screening, treatment, and prophylactic strategies, and (3) potential molecular targets for diet modification as adjuvant therapy to prevent and/or control disease. Finally, the MNS must develop the capacity to conduct independent clinical research. The MNS must be mentored to identify nutritionally relevant clinical problems and to devise testable solutions using clinical research methodology. To our knowledge, few, if any, training programs combine knowledge of food and nutrition science with elements of medical science and practice and with clinical research methodology to create a true translational medical nutrition scientist.

\subsection{Pilot Curriculum to Train the Non-Physician Medical} Nutrition Scientist. Given the dearth of doctoral programs to train the MNS and the pressing need for these professionals to liaise with physicians and researchers, we hypothesized that candidates with doctoral training in nutrition could be trained in the language and practice of medicine and in clinical research methodology at the postdoctoral level. Using R25 funds from the National Institute of Diabetes and Digestive Diseases (NIDDK), we developed and tested an elevenmonth transitional curriculum comprising intensive didactic independent study, guided exposure to clinical medicine, and mentored experience with clinical research strategy and grant writing. Limited training duration precluded the opportunity to conduct research and generate pilot data. However, the Fellows were advised to write up a research proposal that would be appropriate and compatible with the resources of their home institutions. Fellows were also encouraged to undertake subsequent traditional postdoctoral fellowships or to develop collaboration with faculty mentors and conduct the research in their home academic institutions. The basic curriculum created for the NIDDK award focused on obesity 
TABLE 1: Case modules for nutrition, obesity, and comorbidities.

\begin{tabular}{ll}
\hline Critical care & Obese patient with abdominal gunshot wounds and sepsis \\
Endocrinology & Type 1 diabetes, celiac disease, and anemia of chronic disease \\
Metabolic & Type II diabetes, obesity, neuropathy, gout, and sarcopenia \\
Neoplasia & Postmenopausal uterine cancer \\
Gastroenterology & Intestinal permeability in inflammatory bowel disease \\
Liver disease & Nonalcoholic hepatosteatosis (NASH) and metabolic syndrome \\
Cardiology & Atherosclerosis, cardiomyopathy, and congestive heart failure \\
Pulmonary disease & Asthma in an obese young adult \\
Nephrology & Diabetes, arterial calcification, and osteodystrophy in kidney failure \\
Neurology & Alzheimer's disease in a formerly obese man \\
Bone disease & Osteoporosis in a formerly lean elderly man \\
Bariatric surgery & Neurological complications following bariatric surgery \\
\hline
\end{tabular}

Case modules have been developed to illustrate complications of metabolic dysregulations associated with obesity and metabolic syndrome. Patient information with results of imaging and diagnostic testing is presented, followed by a series of questions on the actions of dietary components in the pathophysiology. Resources include an extensive review of the literature linking nutrients with disease pathophysiology, prevention, and treatment.

and its comorbidities. Based on the results of our curriculum development, we propose that this basic curriculum can be modified as a model for specialized medical nutrition training in other specialties, including cancer.

To enhance the candidate's existing knowledge and skills and to remediate any deficits, we instituted the following strategies.

(i) Pathology Based Case Modules. Case modules were integrated into a guided nutritional pathophysiology tutorial that extended throughout the 11-month fellowship. Each module consisted of a two-page scenario describing the patient, complete with relevant laboratory and imaging data, medical and diet history, and results of the physical examination assessment. The scenarios were based on actual or published complex cases and describe not only the presenting symptoms, but also comorbidities and complications. Each case scenario was followed by a literature review setting forth disease processes and potential mechanisms through which nutrients can modify disease pathways. Links were provided to original research reports as well as meta-analyses and consensus reports that evaluated the strength of the evidence currently available. The case modules served two purposes. They served as a nutritional pathophysiology text to supplement the basic pathology text and as links to current medical nutrition therapy recommendations. This information prompted the candidate to develop biological plausibility relating dietary components to the disease processes. Modules served as an evaluation tool to test the candidate's understanding of the material and also revealed unanswered mechanistic questions that the candidate could test in a clinical protocol. Candidates worked through each module independently and presented it to program faculty prior to undertaking the relevant clinical observership. A list of case modules created for the NIDDK award is found in Table 1.

(ii) Introduction to Medicine. Introduction to medical practice was provided in two venues. Initially, candidates reviewed an online tutorial on the rationale and methodology for medical diagnosis and treatment. This resource provided information on diagnostic paradigms and current treatment modalities in an organ system format. Following completion of the tutorial, candidates entered the initial Endocrinology Observership where they received "Hands-on" introduction to medical diagnosis and treatment provided by program faculty. Fellows were also trained in selected use of the online institutional medical records system. A current textbook of medicine complete with supplemental online material was provided to each candidate for detailed discussion of medical considerations and procedures.

(iii) Clinical Observerships. Fellows completed a series of 4to 6-week rotations in selected medical disciplines at the level of a third-year medical student. Clinical mentors provided a "hands on" introduction to patient care in their discipline and supervised candidates throughout the observership. Fellows observed physical, laboratory, and imaging diagnostic methods used in each discipline. Trainees had access to patient information, participated in staff rounds and seminars, and observed selected consults and procedures during their rotation. In the course of each observership, candidates presented actual, nutritionally informative cases to the clinical staff in rounds and seminar settings and discussed diet modification appropriate to each patient. Candidates were asked to respond to staff questions on both the biological plausibility and clinical applicability of a proposed targeted modification. Observerships were evaluated by program faculty using interim and exit interviews with the candidate and review of the candidate's journal, containing data on patients seen, procedures observed, rounds attended, and the like. Each mentor was asked to comment on the fellowship and to assess candidate performance in the observership; see typical observership schedules in Table 2 .

(iv) Clinical Research Mentoring. At the onset of training and prior to undertaking clinical observerships, candidates were required to complete online training in responsible conduct of clinical research. Over the course of the clinical observerships, candidates were expected to identify 
TABLE 2: Observership sites.

Endocrinology and nutrition support
Gastroenterology
General medicine
Geriatrics
Endocrinology
Cardiology
Pulmonary and intensive care
Nephrology
Bariatric surgery
Fellows participate in rotations for four to six weeks, depending on the
site. Mentors in each site have agreed to supervise Fellows at the level of
the third-year medical student. Fellows attend rounds and conferences,
observe procedures, participate in case discussions, and identify clinical
problems with nutritional implications. The Fellow selects one or more of
these problems for development as a clinical research protocol.

nutritionally related clinical problems, develop a biological plausibility for a selected study, explore appropriate clinical and/or basic research methodology, and develop a protocol suitable for submission to a funding agency. To facilitate these activities, candidates met with multiple clinical and basic research mentors to discuss ongoing projects and become familiar with research methodology used by the mentor. The candidate was encouraged to develop collaborations with mentors in his/her area of interest. It is expected that the network of mentor collaborations developed will extend past the fellowship duration and help develop the candidate's research career.

\section{Results and Discussion}

To date, we have trained four postdoctoral fellows, all of whom hold university faculty positions in nutrition. Extensive formative evaluation of candidate progress through the curriculum revealed several barriers; we have identified and addressed barriers as follows.

(i) Prior Training. All candidates completed undergraduate science prerequisites and graduate nutrition science training. While they had designed and conducted independent doctoral research, several of their research topics were narrowly focused in some area of bench nutrition science. None of the candidates had taken a formal course in pathology. Some candidates ( 3 of 4 ) were credentialed as registered dietitians; however, neither doctoral training nor clinical knowledge required for dietetic credentialing includes comprehensive study of nutritional pathophysiology or clinical medicine. None of the candidates had undertaken an observership in clinical medicine. Candidates indicated that their greatest difficulty was in following the medical considerations and rationale for treatment of specific patients.

To address this barrier, we formalized the nutritional pathophysiology course to extend through the duration of the fellowship. In response to formative evaluation, we now require candidates to complete and present the corresponding case module prior to undertaking the clinical observership. We have also required that the online introduction to medicine be completed prior to entering the Endocrinology Observership with its "hands on" introduction to medicine segments. Because we found that the medical terminology and abbreviations posed a challenge to some candidates, we introduced weekly interactive sessions for the candidate with faculty mentors; these sessions have significantly enhanced the Fellow's learning.

(ii) Candidate Recruitment. R25E funds are awarded for program development, not trainee support. Although trainees receive a stipend to defray part of their living expenses, this award does not constitute a salary as does the traditional postdoctoral fellowship. Thus, although we received applications from a number of highly qualified and enthusiastic newly minted Ph.D. applicants, all found the stipend insufficient and declined to participate in the training. Our short-term solution to this problem was to recruit candidates from nutrition faculty supported by their institutions during their sabbatical year. Prospective candidates were recruited from members of the Academy of Nutrition and Dietetics, specifically from the dietetic educators practice group. This solution posed further problems as delineated below.

(iii) Recency of Research. Of the four candidates trained, two candidates had undertaken heavy teaching and administrative duties for several years after doctorate. Because these responsibilities had prevented the candidates from keeping abreast of ongoing research in nutrition and medicine, the heavy focus on nutrition pathology, medicine, and clinical research in our curriculum presented a challenge. This barrier appears to have been surmounted by our final candidate who obtained her academic appointment prior to completing her doctoral research. This permitted her to take a sabbatical immediately after obtaining her doctorate. Although she had some of the knowledge gaps indicated above, her research skills were more current and her progress both in the clinical observerships and clinical research development appeared to be much more productive than her predecessors. This final candidate was able to identify several clinical problems in need of research; she is presently developing one of these problems with a faculty mentor and will use it for her grant proposal. These observations support the necessity of full-time candidate funding for the one-year transition postdoctoral fellowship.

(iv) Candidate Credentialing. A major barrier for some candidates appeared to be a functional dichotomy between the scope of practice of a registered dietitian and the requirements of the MNS curriculum. Candidates, who were credentialed as registered dietitians (RD) and had spent several years teaching dietetics, attempted to apply existing medical nutrition guidelines to a clinical problem. In contrast, the goal of MNS training is to train the Fellow to identify a nutritionrelated clinical problem and develop a testable protocol to generate new knowledge. The final candidate also struggled with this dichotomy, but because she had recently completed 
her doctorate, she was better able to identify potential research questions in the clinical observerships. It should be noted that clinical mentors were very happy to access the dietetic knowledge and skills of the Ph.D., RD Fellows.

(v) Short Duration of Training. Most traditional postdoctoral fellowships are at least two years in duration, during which the candidate is required to devise a research project and generate pilot data for further research. While the one-year duration of our transition training paradigm could be considered a barrier, we have determined it is a unique solution. By designating the fellowship as "transitional," we reinforce the concept that this first postdoctoral year could almost be considered an extension of doctoral training. The MNS training provides clinical knowledge and experiences not generally available in doctoral nutrition programs. We expect that completion of the transitional year will ideally position the candidate to seek a traditional research fellowship or academic position in which he/she will conduct substantive translational research.

\section{Conclusions}

From these pilot data we conclude that it is possible to efficiently train a MNS candidate with the competencies delineated above. It is critical that candidates have completed solid basic science coursework at the undergraduate level, have undertaken doctoral level nutrition and medical science training, and have obtained a working knowledge of all aspects of food and nutrition. During the transitional year, the candidates must have access to expert mentors in medical nutrition, medical specialties, and clinical research. Candidates must be funded with a living salary to assure that highly motivated, recent Ph.D. graduates will apply. With these elements in place, we propose that our intensive oneyear postdoctoral fellowship curriculum can provide didactic training and clinical experiences sufficient to transition the candidate into translational clinical research. We expect that, following MNS training, graduates will function as translational researchers, educators, and expert consultants to the medical, research, and public health communities.

\section{Conflict of Interests}

The authors declare that there is no conflict of interests regarding the publication of this paper.

\section{Authors' Contribution}

Drs. Ettinger, Nasser, Engelson, Albu, Hashim, and Pi-Sunyer made major contributions to the overall research plan and project oversight; Dr. Ettinger wrote the paper and Dr. PiSunyer had primary responsibility for the final content.

\section{Acknowledgments}

Program development was supported by an R25E grant (2008) from NIDDK (PAR06-554): Innovative Translational Medical Nutrition Training for M.D. and Ph.D. Trainees and the NYONRC fund no. SL45003322, Grant no. DK080664. The authors also acknowledge the contributions of members of the New York Obesity Nutrition Research Center for mentoring Fellows in the rationale and methodology of their current research interests and the physicians at the St. Luke's Roosevelt Hospital Center for training the Fellows in the language and practice of their diverse medical disciplines. Finally, they acknowledge the contributions of their Fellows for providing insight into effective strategies for program development.

\section{References}

[1] Hippocrates, On Ancient Medicine-Part 14, 400 BC.

[2] J. A. Baur, K. J. Pearson, N. L. Price et al., "Resveratrol improves health and survival of mice on a high-calorie diet," Nature, vol. 444, no. 7117, pp. 337-342, 2006.

[3] S. P. Weisberg, R. Leibel, and D. V. Tortoriello, "Dietary curcumin significantly improves obesity-associated inflammation and diabetes in mouse models of diabesity," Endocrinology, vol. 149, no. 7, pp. 3549-3558, 2008.

[4] J. F. Leikert, T. R. Räthel, P. Wohlfart, V. Cheynier, A. M. Vollmar, and V. M. Dirsch, "Red wine polyphenols enhance endothelial nitric oxide synthase expression and subsequent nitric oxide release from endothelial cells," Circulation, vol. 106, no. 13, pp. 1614-1617, 2002.

[5] H. Schroeter, C. Heiss, J. Balzer et al., “(-)-epicatechin mediates beneficial effects of flavanol-rich cocoa on vascular function in humans," Proceedings of the National Academy of Sciences of the United States of America, vol. 103, no. 4, pp. 1024-1029, 2006.

[6] J. A. Milner, "Incorporating basic nutrition science into health interventions for cancer prevention," Journal of Nutrition, vol. 133, no. 11, pp. 3820S-3826S, 2003.

[7] J. W. Finley, "Proposed criteria for assessing the efficacy of cancer reduction by plant foods enriched in carotenoids, glucosinolates, polyphenols and selenocompounds," Annals of Botany, vol. 95, no. 7, pp. 1075-1096, 2005.

[8] S. Egert and G. Rimbach, "Which sources of flavonoids: complex diets or dietary supplements?" Advances in Nutrition, vol. 2, no. 1, pp. 8-14, 2011.

[9] K. M. Munir, S. Chandrasekaran, F. Gao, and M. J. Quon, "Mechanisms for food polyphenols to ameliorate insulin resistance and endothelial dysfunction: therapeutic implications for diabetes and its cardiovascular complications," American Journal of Physiology: Endocrinology and Metabolism, vol. 305, no. 6, pp. E679-E686, 2013.

[10] I. Sekirov, S. L. Russell, L. C. M. Antunes, and B. B. Finlay, "Gut microbiota in health and disease," Physiological Reviews, vol. 90, no. 3, pp. 859-904, 2010.

[11] V. L. W. Go, R. R. Butrum, and D. A. Wong, "Diet, nutrition, and cancer prevention: the postgenomic era," Journal of Nutrition, vol. 133, no. 11, supplement 1, pp. 3830S-3836S, 2003.

[12] J. B. German, M. A. Roberts, L. Fay, and S. M. Watkins, "Metabolomics and individual metabolic assessment: the next great challenge for nutrition," Journal of Nutrition, vol. 132, no. 9, pp. 2486-2487, 2002.

[13] D. C. Heimburger, "Training and certifying Physician Nutrition Specialists: the American Board of Physician Nutrition Specialists (ABPNS)," The American Journal of Clinical Nutrition, vol. 83, no. 4, pp. 985S-987S, 2006. 
[14] D. C. Heimburger, S. A. McClave, L. M. Gramlich, and R. Merritt, "The intersociety professional nutrition education consortium and American board of physician nutrition specialists: what have we learned?" Journal of Parenteral and Enteral Nutrition, vol. 34, no. 6, supplement, pp. 21S-29S, 2010.

[15] T. B. van Itallie, "The 'pure' clinical nutritionist: an endangered species," The American Journal of Clinical Nutrition, vol. 30, no. 12, pp. 1929-1934, 1977.

[16] S. A. McClave, J. I. Mechanick, B. Bistrian et al., "What is the significance of a physician shortage in nutrition medicine?" Journal of Parenteral and Enteral Nutrition, vol. 34, no. 6, supplement, pp. 7S-20S, 2010.

[17] D. S. McLaren, "Nutrition in medical schools: a case of mistaken identity," The American Journal of Clinical Nutrition, vol. 59, no. 5, pp. 960-963, 1994.

[18] M. E. Shils, "National dairy council award for excellence in medical and dental nutrition education lecture, 1994: nutrition education in medical schools-the prospect before us," American Journal of Clinical Nutrition, vol. 60, no. 4, pp. 631-638, 1994.

[19] D. S. McLaren, "The need for physician nutrition specialists," American Journal of Clinical Nutrition, vol. 62, no. 2, pp. 446447, 1995.

[20] L. H. Allen, M. E. Bentley, S. M. Donovan, D. M. Ney, and P. J. Stover, "Securing the future of nutritional sciences through integrative graduate education," Journal of Nutrition, vol. 132, no. 4, pp. 779-784, 2002. 

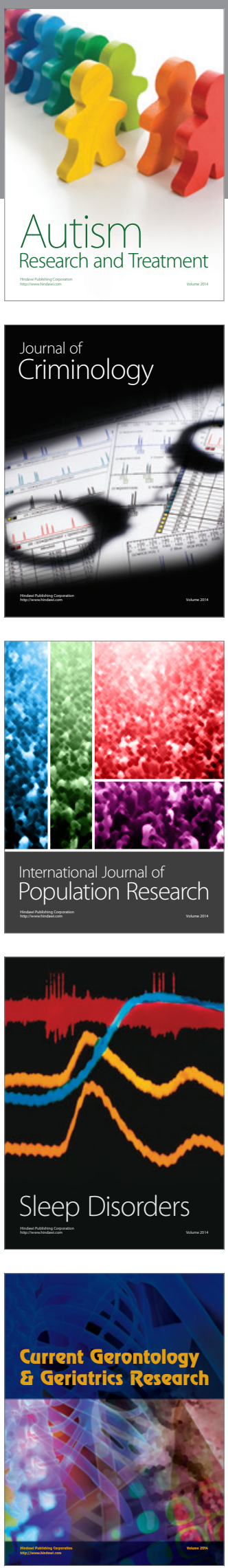
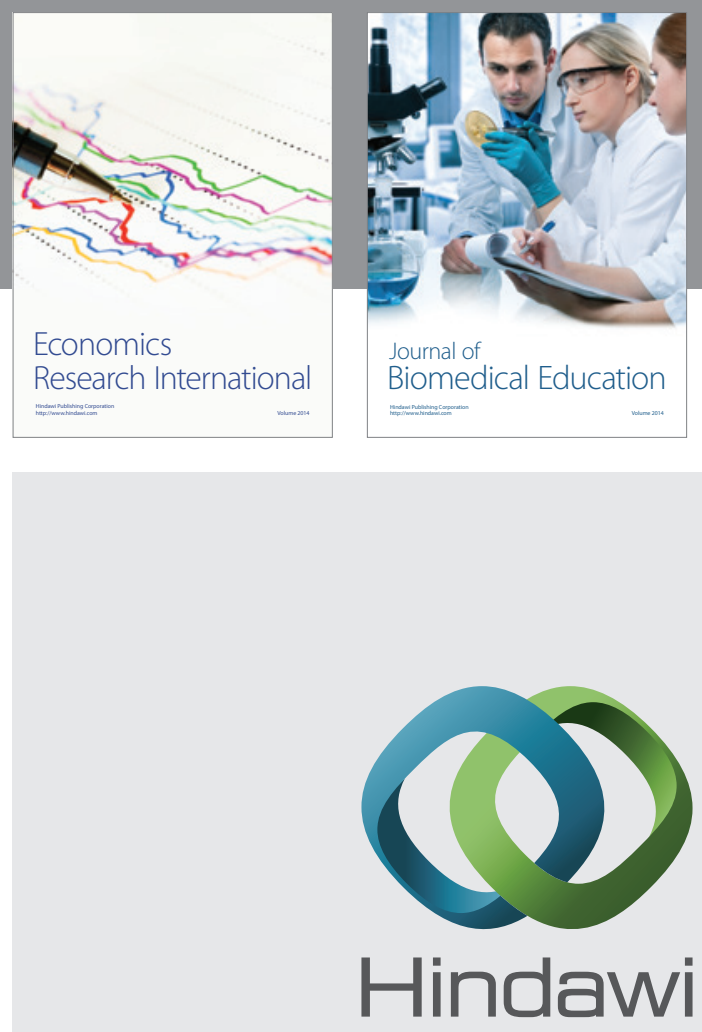

Submit your manuscripts at

http://www.hindawi.com
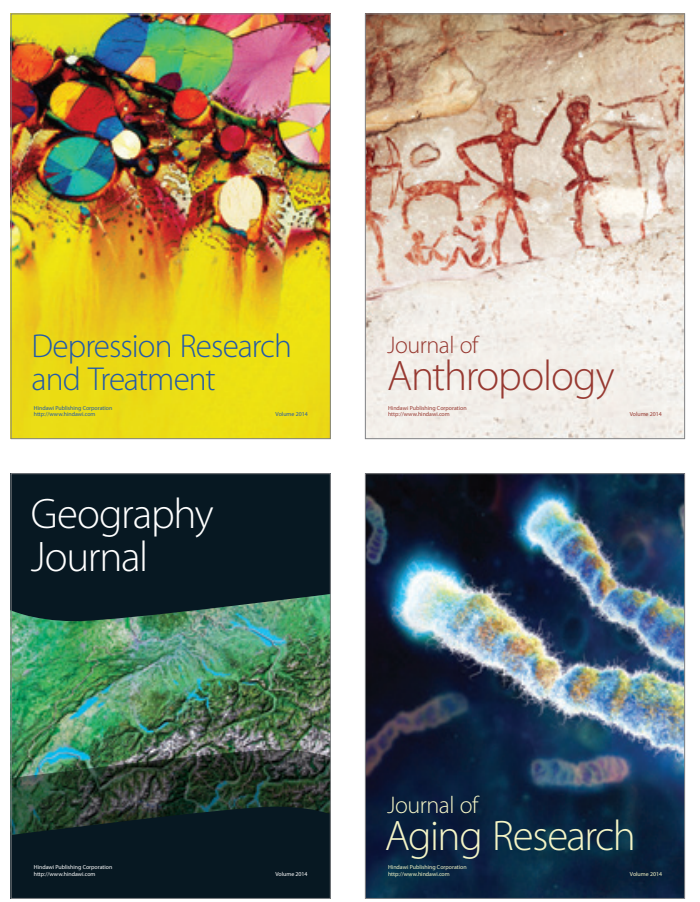
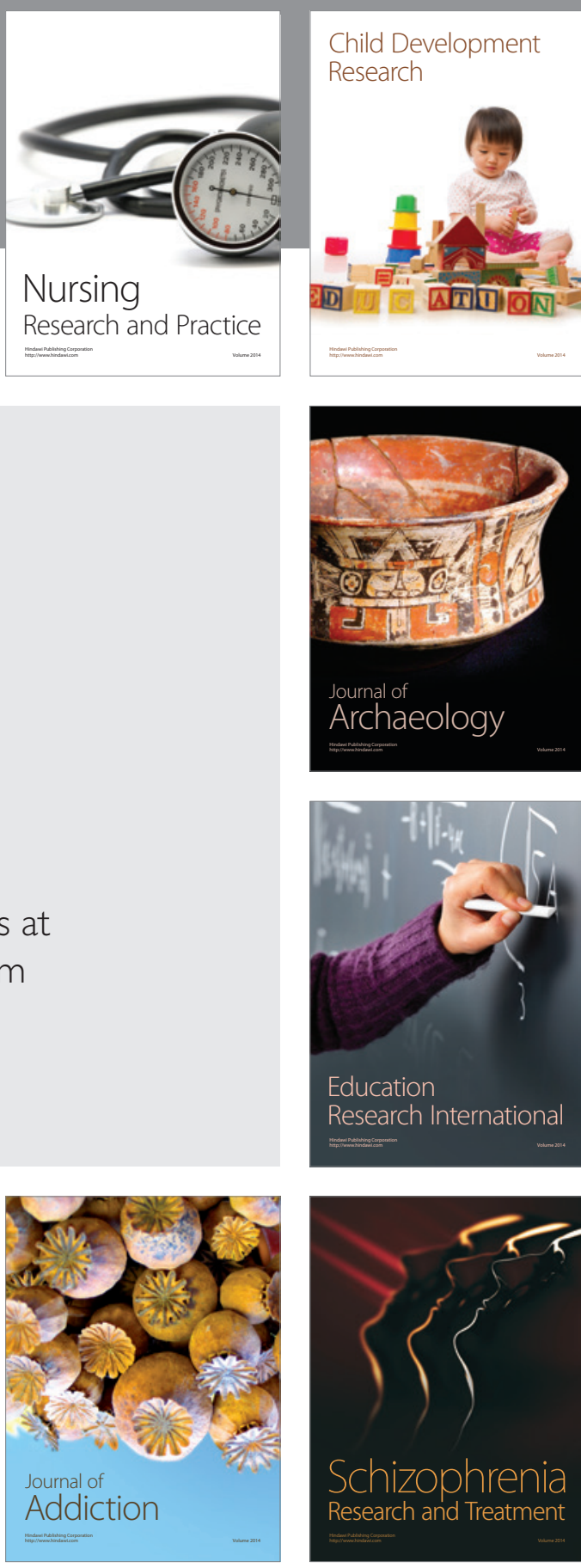

(D)
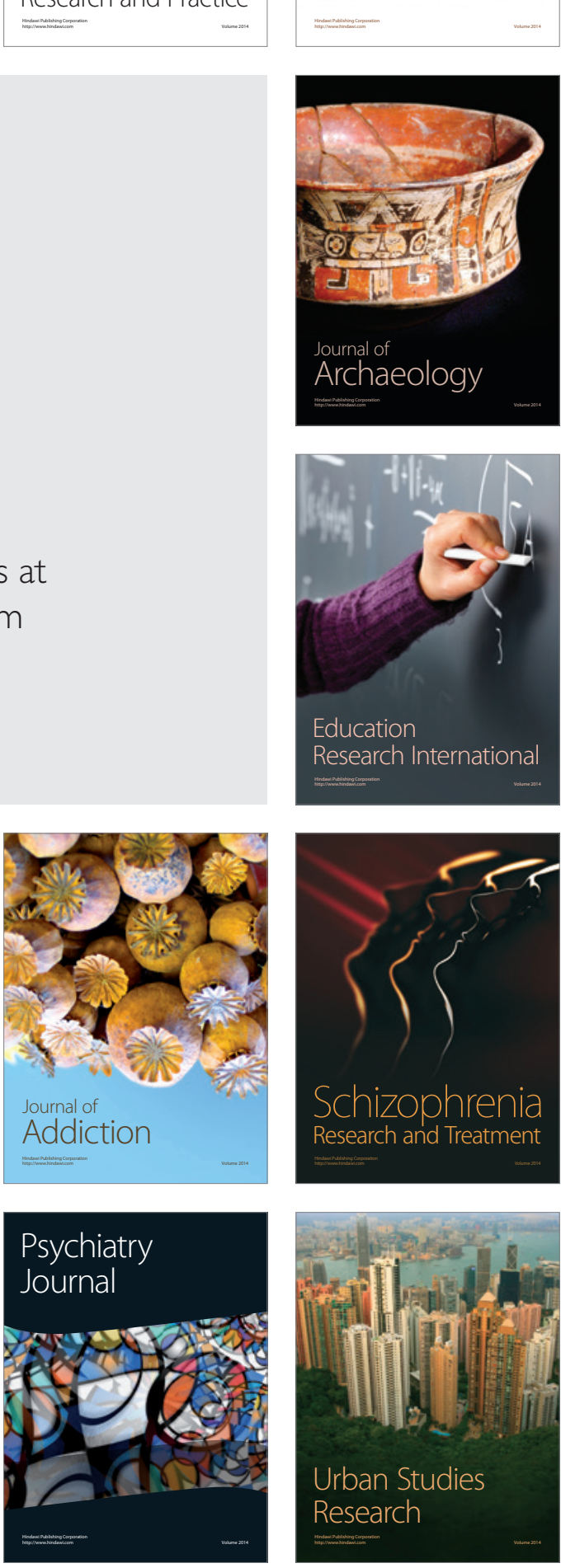